\title{
An extended cohesive damage model with a length scale in fracture analysis of adhesively bonded joints
}

\author{
J Chen \\ Faculty of Technology, University of Portsmouth \\ Portsmouth PO1 3AH, UK \\ Tel: 02392842427 \\ Email: jiye.chen@port.ac.uk
}

\begin{abstract}
This paper introduced an extended cohesive damage model (ECDM) in fracture analysis of adhesively bonded joints. A length scale of pore size at joints was introduced in the accumulation of damages in the proposed ECDM. This micro damage scale based model was used to build up an extended cohesive finite element modelling of adhesively bonded joints at a macro level. An adhesive damage law (ADL) was used to simulate debonding at interfaces. A cohesive damage law (CDL) was proposed to simulate internal cohesive cracks. These two micro damage laws firstly considered the length scale influence from the interfacial pores and internal particles. These ECDM models were used to deal with the stress discontinue problems at interfaces or internal materials. As an example, an adhesively bonded bone-cement joint was investigated in this paper. The failure mechanism of bone-cement joint under tension, with different bonding qualities, was studied using the developed ECDM models. This investigation indicated that the effect of bonding quality on the loading capacity of bone-cement joint can be studied properly by the ECDM with a length scale of interfacial pore size. This ECDM based damage modelling technique supplied an effective and efficient approach in cohesive and adhesive damage analysis.
\end{abstract}

Key Words: Extended cohesive damage model, length scale modelling, adhesively bonded joints, adhesive failure analysis 


\section{Nomenclature}

ECDM: Extended cohesive damage model

ABJ: Adhesively bonded joints

CZM: Cohesive zone model

XFEM: Extended finite element method

ADL: Adhesive damage law

CDL: Cohesive damage law

U: Enriched displacement field

$\phi$ : A standard basis function $\psi_{\mathrm{j}}$ is the enriched function

$\mathbf{N}$ : Shape function as the standard

$\mathbf{H}_{\Gamma \mathrm{d}}$ : Heaveside function

$\mathbf{a}$ :Standard degrees of freedom

b: Enriched degrees of freedom

$\Omega$ : Volume of body

$\boldsymbol{\sigma}$ : Cauchy stress tensor

$\mathbf{u}^{s}$ : Standard part of displacement

$\mathbf{u}^{e}$ : Enriched part of displacement

B: Matrix of spatial derivatives of $\mathbf{N}$ in the geometric equation

D: Tangent material stiffness matrix

$\mathbf{K}_{\Gamma \mathrm{d}}$ : Tangent interface stiffness matrix

$\mathbf{f}_{\text {ext }}$ and $\mathbf{f}_{\text {int }}$ are the external and internal forces

$\lambda$ : Pore size at interface between adhesives and adherents

$\mathrm{K}_{0}(\lambda)$ : Initial interface stiffness

$\sigma_{\mathrm{a}}(\lambda)$ : Interface strength

$\varepsilon_{0}(\lambda)$ : Initial damage relative displacement

$\beta$ : Particle size in adhesives

$\mathrm{E}(\beta)$ : Cohesive module

$\varepsilon_{0}(\beta)$ : Initial damage strain

$\sigma_{t}(\beta)$ : Cohesive strength

d: Damage scale

$\gamma$ : Damage coupling factor

$\mu$ : Mixed damage ratio is taken

A: Interface property constant matrix

$\mathrm{G}_{\mathrm{c}}(\lambda / \beta)$ : Fracture toughness 


\section{Introduction}

The study of the fracture behaviour of adhesively bonded joints (ABJ) such as bone-cement joints is essential for the design of implant in biomedical engineering. Previous work investigated the failure mechanism of bone-cement joints with consideration of an averaged bonding quality at interface using a standard cohesive zone model (CZM) [1-3] to study the fracture at bone-cement interfaces. However, the effect of length scale of pore size at interface of joints has not been studied yet. The cohesive zone model was applied widely in the last decade, it has been successfully applied for simulation of many engineering material or structural problems such adhesives debonding, biomedical joint's fractures [4-5], delamination in fibre composites [6-17], etc. CZM model is an interfacial traction-separation law based model to simulate crack propagation. The interfacial fracture toughness, strength and stiffness are used to form the cohesive damage law (CDL). It should be noticed that the bonding quality of $\mathrm{ABJ}$ s is affected by the pores at the interface between adhesives and adherents. Thus interfacial fracture toughness, strength and stiffness should be influenced by the pore size at interfaces. It is hardly to be sure the standard CZM model can reflect such micro effects on interface damage behavour. Previous work [1-5] only supplied approaches to study over all behaviour of adhesively bonded interfaces using averaged interfacial parameters. It should be noticed that studying the detailed effects of micro pores at general ABJs on their damage mechanism is important in investigating a way to enhance their damage resilience. Therefore, a new damage model with the effects of length scale of micro pores is required to build up a macro modelling in progressive damage analysis of ABJs. In general, the behaviour of ABJs is not only affected by the bonding strength of interface but also affected by cohesive strength. A heterogeneous adhesive (Xu et al. 2003 and White et al., 2001) [1, 2] has been developed to improve bonding strength of ABJs. The heterogeneous adhesive usually contains micro particles to change material internal loading transferring and storing deformed energy [2]. However, the size of micro particles contained in adhesives certainly influences the adhesive strength in ABJs. Thus, a new damage model with the effects of length scale of micro particles is also required in simulation of progressive damages of ABJs.

The standard finite element method (FEM) has an unavoidable difficulty in the simulation of damage / crack propagation as it requires re-meshing of the cracked geometry. Additionally, in order to capture the true stress and strain field around the crack tip, mesh refinement is mandatory. In the last decade, a fracture mechanics based standard CZM model was successfully used in the simulation of interfacial separation, for example delamination in composites. However, this model 
is hardly to simulate the entire failure process because it relies on an already-known crack path. Therefore, either standard FEM technique or standard CZM model is not a feasible modelling tool for the prediction of multiple crack propagation in ABJs and composite structures.

The publication of an initial extended finite element method (XFEM) for the automatic simulation of crack propagation of homogeneous materials [A Ahmed, et al. 2009] (20) and [T.P. Fries, 2011] (21) represented a significant breakthrough in the predictive computation for damage, fracture, fatigue, corrosion, erosion, diffusion, interaction, etc. Since 2009 the XFEM has draw a lot of attention in the academia of computational mechanics for solids and structures with significant discontinuities. The scientific innovation within XFEM is the potential to accurately consider damage evolution in heterogeneous materials with structural discontinuities, such as cracks, defects or imperfections using specific enrichment functions. These mathematical enrichment functions will extend the specific capability of standard FEM for addressing the problem of stress singularity at the crack tip, and for addressing existing cracks. The standard FEM supplies a numerical domain of object for a continuous analysis; the XFEM based on the FEM domain supplies an extended domain for a discontinuous analysis of the same object. The XFEM can set up the failure criteria to determine crack initiation and growth, automatically calculate the direction of crack growth. Simulated cracks can either go through any interfaces between elements or split elements. Therefore, the XFEM can simulate the real crack path in complex structures, thus corresponding damage tolerance can be predicted precisely. Currently, most published research of XFEM has focused on the simulation of fracture growth in homogeneous materials. It should be noticed that it is still a significant challenge for using XFEM in simulation of multiscale damage, multicrack propagation in ABJs and composite structures. Because a set of different enrichment functions and a set of different failure criteria are required to overcome the discontinue problems with different cracks in complex ABJs and composites. Also, determination of direction of crack propagation would vary according to different types of damage / crack including crack splitting.

In this investigation, a new damage modelling technique ECDM combined XFEM with CZM, was introduced in this paper and used in the investigation of the length scale damage analysis of ABJs. This developed micro ECDM was used to build up a macro finite element modelling of ABJs. Both pores at interfaces and internal particles were included as length scale effects in ECDM. Corresponding adhesive damage law (ADL) and CDL firstly reflected the length scale. The coupled damage effects in three fracture modes were also accounted in the developed ECDM. Finally, a length scale damage analysis of a typical $\mathrm{ABJ}$ of bone-cement joint as an example was presented in 
this paper. Previous experimental work [3] gave three different bonding qualities of bone-cement interface, which were represented by three different sizes of micro pores at adhesively bonded interface. The developed ECDM with length scale of pore size was used to investigate the effects of three different interface bonding qualities on the prediction of loading capacity of bone-cement joint under tension.

\section{Brief XFEM formulation}

In the XFEM, a discontinuous displacement enrichment based on a local partition of unity plays a key role to approximate the displacement field of the damage zone [20, 21]. A standard basis function $\phi_{\mathrm{i}}$ related to node $\mathrm{i}(1 \leq \mathrm{i} \leq \mathrm{n})$ forms a partition of unity, i.e.

$$
\sum_{i=1}^{n} \phi_{i}=1
$$

The enriched displacement field $\mathbf{u}$ in the damaged zone can be interpolated as

$$
\mathbf{u}(x)=\sum_{i=1}^{n} \phi_{i}\left(a_{i}+\sum_{j=1}^{m} \psi_{j}(x) b_{i j}\right)
$$

Where, $\mathrm{n}$ is the number of the nodes of element enriched, and $\psi_{\mathrm{j}}$ is the enriched function with $\mathrm{m}$ terms. $x$ is the position of a material point, $a_{i}$ is the standard degrees of freedom and $b_{i j}$ is the enriched degrees of freedom representing the magnitude of the jth term of $\psi \mathrm{j}$ at node $\mathrm{i}$. Considering that the usual element shape function $\mathrm{N}_{\mathrm{i}}$ as the standard basis function to replace $\phi_{\mathrm{i}}$ and also using Heaveside function $\mathrm{H}_{\Gamma \mathrm{d}}$ to replace $\psi_{\mathrm{j}}$ in approximating discontinuity, the displacement filed $\mathbf{u}$ at damaged zone given in equ. 2 can be changed as

$\mathbf{u}(x)=\sum_{i=1}^{n} N_{i} a_{i}+H_{\Gamma d} \sum_{i=1}^{n} N_{i} b_{i}$

Where, the first item is equ. 3 is a standard displacement, the second item is an enriched displacement. Figure 1 shows a body with volume $\Omega\left(\Omega^{+} \cup \Omega^{-}\right)$, which has a boundary condition for both tractions and displacement. The Heaveside function $\mathrm{H}_{\Gamma \mathrm{d}}$ can be defined as 


$$
H_{\Gamma d}=\left\{\begin{array}{cc}
1 & x \in \Omega^{+} \\
-1 & x \in \Omega^{-}
\end{array}\right.
$$

In the Figure 1, the boundary $\Gamma_{\mathrm{u}}$ is restrained by given displacements $\mathbf{u}, \Gamma_{\mathrm{t}}$ is loaded by the external traction $\overline{\mathbf{t}}$ with the exterior normal vector $\mathbf{n}$. $\mathbf{t}$ is the traction at discontinuity $\Gamma_{d}$ with the normal $\mathbf{m}$ directing from $\Omega^{-}$to $\Omega^{+}$. The equilibrium equation on the body without body forces and the corresponding boundary conditions are given as

$$
\begin{aligned}
& \nabla \bullet \boldsymbol{\sigma}=0 \quad x \in \Omega \\
& \boldsymbol{\sigma} \bullet \mathbf{m}=\mathbf{t} \quad x \in \Gamma_{d} \\
& \boldsymbol{\sigma} \bullet \mathbf{n}=\overline{\mathbf{t}} \quad x \in \Gamma_{t} \\
& \mathbf{u}=\overline{\mathbf{u}} \quad x \in \Gamma_{u}
\end{aligned}
$$

Where, $\mathbf{u}$ is the displacement vector, $\boldsymbol{\sigma}$ denotes the Cauchy stress tensor. Using the BubnovGalerkin approach, the weak form of the equilibrium equation can be obtained by introducing admissible displacement variations $\delta \mathbf{u}$, as

$$
\int_{\Omega} \delta \mathbf{u} \bullet(\nabla \bullet \boldsymbol{\sigma}) d \mathbf{\Omega}=0
$$

In equ. 6 , the $\delta \mathbf{u}$ is chosen from the same shape function used for constructing the displacement field $\mathbf{u}$ given in equ. 7.

$$
\delta \mathbf{u}=\delta \mathbf{u}^{s}+H_{\Gamma_{d}} \delta \mathbf{u}^{e}=\mathbf{N} \delta \mathbf{a}+H_{\Gamma_{d}} \mathbf{N} \delta \mathbf{b}
$$

Where, $\mathbf{u}^{s}$ and $\mathbf{u}^{e}$ are the standard and enriched part of displacement respectively. Bring equ.7 into equ. 6 results

$$
\int_{\Omega}\left[\boldsymbol{\delta} \mathbf{u}^{s} \bullet(\nabla \bullet \boldsymbol{\sigma})+H_{\Gamma_{d}} \delta \mathbf{u}^{e}(\nabla \bullet \boldsymbol{\sigma})\right] d \boldsymbol{\Omega}=0
$$

The governing equation of the XFEM can be obtained integrating by parts and applying the Gaussian divergence theorem as shown in equ. 9 [23]. 


$$
\left\{\begin{array}{c}
\int_{\Omega} \nabla \delta \mathbf{u}^{s}: \boldsymbol{\sigma} d \Omega=\int_{\Gamma_{t}} \delta \mathbf{u}^{s} \bullet \overline{\mathbf{t}} d \Gamma \\
\int_{\Omega} \nabla \delta \mathbf{u}^{e}: \boldsymbol{\sigma} d \Omega+\int_{\Gamma_{d}} \delta \mathbf{u}^{e} \bullet \overline{\mathbf{t}} d \Gamma=\int_{\Gamma_{t}} H_{\Gamma_{d}} \delta \mathbf{u}^{e} \bullet \overline{\mathbf{t}} d \Gamma
\end{array}\right.
$$

In the equ. 9, the CDL was used together with additional equations to express the mechanical behavior of the interface $\Gamma_{\mathrm{d}}$. The strain field in an element can be obtained after using equ. 3 differentiation as

$\boldsymbol{\varepsilon}=\nabla^{s} \mathbf{u}=\mathbf{B a}+H_{\Gamma_{d}} \mathbf{B b}$

Where, the matrix $\mathbf{B}$ contains the spatial derivatives of $\mathbf{N}$ in the geometric equation and $\nabla^{\mathrm{s}}$ means the symmetric part only in terms of the small-deformation assumption. The constitutive relationship between the stress increment and the strain increment can be expressed as

$\nabla \boldsymbol{\sigma}=\mathbf{D}\left(\mathbf{B} \nabla \mathbf{a}+H_{\Gamma_{d}} \mathbf{B} \nabla \mathbf{b}\right)$

Where, $\mathbf{D}$ is the tangent material stiffness matrix, the traction at $\Gamma_{\mathrm{d}}$ can be written as a function of the discontinuity $\mathbf{u}^{\mathrm{e}}$. In the cohesive zone, the CDL can be generally expressed as a nonlinear function of separation $\mathbf{u}^{\mathrm{e}}$, i.e. $\sigma=\sigma\left(\mathbf{u}^{\mathrm{e}}\right)$. Its differentiation results

$\Delta \boldsymbol{\sigma}=\frac{\partial \boldsymbol{\sigma}}{\partial \mathbf{u}^{e}} \Delta \mathbf{u}^{e}=\mathbf{K}_{\Gamma d} \Delta \mathbf{u}^{e}=\mathbf{K}_{\Gamma d} \mathbf{N} \Delta \mathbf{b}$

In equ. $12, \mathbf{K}_{\Gamma \mathrm{d}}=\partial \sigma / \partial \mathbf{u}^{\mathrm{e}}$ is the tangent stiffness matrix between the increment of the traction and the increment of the separation at $\Gamma_{\mathrm{d}}$. Using equs. 10 and 11, the governing equation for the XFEM given in equ. 9 can be written by inserting the discredited form of the variation of the displacement field as

$$
\left\{\begin{array}{c}
\int_{\Omega}(\mathbf{B} \delta \mathbf{a})^{T} \mathbf{D}\left(\mathbf{B a}+H_{\Gamma_{d}} \mathbf{B b}\right) d \Omega=\int_{t}(\mathbf{N} \delta \mathbf{a})^{T} \mathbf{t} d \Gamma \\
\int_{\Omega}(\mathbf{B} \delta \mathbf{b})^{T} \mathbf{D}(\mathbf{B a}+\mathbf{B} \mathbf{b}) d \Omega+\int_{\Gamma_{t}}(\mathbf{N} \delta \mathbf{b})^{T} \mathbf{t} d \Gamma=\int_{\Gamma_{t}} H_{\Gamma_{d}}(\mathbf{N} \delta \mathbf{b})^{T} \overline{\mathbf{t}} d \Gamma
\end{array}\right.
$$


The Newton-Raphson scheme was used solve independent unknowns $\mathbf{a}$ and $\mathbf{b}$ independently since a set of nonlinear equations after discretisation. Considering equs. 11 to 13 , the incremental finite element equations are given in the form as below.

$$
\left[\begin{array}{ll}
\mathbf{K}_{a a} & \mathbf{K}_{a b} \\
\mathbf{K}_{b a} & \mathbf{K}_{b b}
\end{array}\right]\left\{\begin{array}{l}
\Delta \mathbf{a} \\
\Delta \mathbf{b}
\end{array}\right\}=\left\{\begin{array}{l}
\mathbf{f}_{a e x t} \\
\mathbf{f}_{b e x t}
\end{array}\right\}-\left\{\begin{array}{l}
\mathbf{f}_{a \mathrm{int}} \\
\mathbf{f}_{b \mathrm{int}}
\end{array}\right\}
$$

Where, the definition of terms is given below.

$$
\left\{\begin{array}{c}
\mathbf{K}_{a a}=\int_{\Omega} \mathbf{B}^{T} \mathbf{D} \mathbf{B} d \Omega \\
\mathbf{K}_{a b}=\mathbf{K}_{b a}=\int_{\Omega} \mathbf{B}^{T} \mathbf{D} \mathbf{B} d \Omega \\
\mathbf{K}_{b b}=\int_{\Omega^{+}} \mathbf{B}^{T} \mathbf{D B} d \Omega+\int_{\Gamma_{d}} \mathbf{N}^{T} \mathbf{K}_{\Gamma d} \mathbf{N} d \Gamma \\
\mathbf{f}_{a e x t}=\int_{\Gamma_{t}} \mathbf{N}^{T} \mathbf{t} d \Omega \\
\mathbf{f}_{b e x t}=\int_{\Gamma_{t}} H_{\Gamma_{d}} \mathbf{N}^{T} \overline{\mathbf{t}} d \Omega \\
\mathbf{f}_{a \text { int }}=\int_{\Omega} \mathbf{B}^{T} \boldsymbol{\sigma} d \Omega \\
\mathbf{f}_{b \text { int }}=\int_{\Omega^{-}} \mathbf{B}^{T} \boldsymbol{\sigma} d \Omega+\int_{\Gamma_{d}} \mathbf{N}^{T} \mathbf{t} d \Gamma
\end{array}\right.
$$

Where, $\mathbf{f}_{\text {ext }}$ and $\mathbf{f}_{\text {int }}$ are the external and internal forces, respectively. $\Delta \mathbf{a}$ and $\Delta \mathbf{b}$ denote the incremental displacements at node. Equs. 1 to 15 were used to form the XFEM formulation in implementation.

It should be noticed that the first item in equ. 3 as standard displacement approximation was used in the whole domain of body, the second item was only applied on the selected elements or nodes which require enrichments to reflect discontinues. Therefore, the second item appears only in the case when some elements have been damaged. The ECDM was used as criteria to judge the damage propagation based on the continue displacement field with first item in the equ. 3, when ECDM is satisfied by some elements then the second item will be applied to penalize damage elements in reflecting discontinuity using the Heaveside function. 


\section{Adhesive and cohesive damage laws}

A bilinear material softening law is one of standard cohesive damage laws was introduced by previous researchers [4-17] and pasted below as shown in equ. 16 for extending its capacity in this investigation.

$\sigma_{j}(\varepsilon)=\left\{\begin{array}{rcc}K_{j 0} \varepsilon_{j} & \text { if } & \varepsilon_{j} \leq \varepsilon_{j 0} \\ (1-d) K_{j 0} \varepsilon_{j} & \text { if } & \varepsilon_{j 0} \leq \varepsilon_{j}<\varepsilon_{j c}, \quad j=I, I I, I I I \\ 0 & \text { if } & \varepsilon_{j} \geq \varepsilon_{j c}\end{array}\right.$

Where, I, II and III regard three individual damage modes respectively. The initial interfacial stiffness $\mathrm{K}_{\mathrm{j} 0}(\mathrm{j}=\mathrm{I}, \mathrm{II}, \mathrm{III})$ in equ. 16 is determined by the interfacial strength $\sigma_{\mathrm{ja}}$ and initial damage relative displacement $\varepsilon_{\mathrm{j} 0}$ as $\mathrm{K}_{\mathrm{j} 0}=\sigma_{\mathrm{ja}} / \varepsilon_{\mathrm{j} 0}(\mathrm{j}=\mathrm{I}, \mathrm{II}, \mathrm{III})$. When equ. 16 is used to simulate interfacial damage at adhesive joint with pores at bonded interfaces shown in Figure 2, the pores effect should be accounted into a modified equation called ADL shown in equ. 17. Note the $\mathrm{K}_{\mathrm{jo}}$ and $\varepsilon_{\mathrm{j}}$ in equ .17 are equivalent to $\mathrm{K}_{\Gamma \mathrm{d}}$ and $\mathrm{u}^{\mathrm{e}}$ in equ. 12.

$\sigma\left(\varepsilon_{j}, \lambda\right)_{j}=\left\{\begin{array}{cccc}K(\lambda)_{j 0} \varepsilon_{j} & \text { if } & \varepsilon_{j}<\varepsilon(\lambda)_{0 j} \\ \left(1-d(\lambda)_{j}\right) K(\lambda)_{j 0} \varepsilon_{j} & \text { if } \quad & \varepsilon(\lambda)_{0 j} \leq \varepsilon_{j}<\varepsilon(\lambda)_{c j} \\ 0 & \text { if } & \varepsilon_{j} \geq \varepsilon(\lambda)_{c j}\end{array}\right.$

Where, the pore size $\lambda$ at interface between adhesives and adherents was included as the length scale in the ADL, which consists of functions for initial stiffness $K(\lambda)_{j 0}$, initial damage relative displacement $\varepsilon_{0 \mathrm{j}}$, critical damage relative displacement $\varepsilon_{\mathrm{cj}}$ and interfacial damage scale $\mathrm{d}(\lambda)_{\mathrm{j}}$. The subscript $\mathrm{j}$ varies in terms of three fracture mode I, II and III.

When equ. 16 is used to simulate internal damage within cohesive with particles shown in Figure 2, the particle effect should be accounted into an extended equation called CDL shown in equ. 18 .

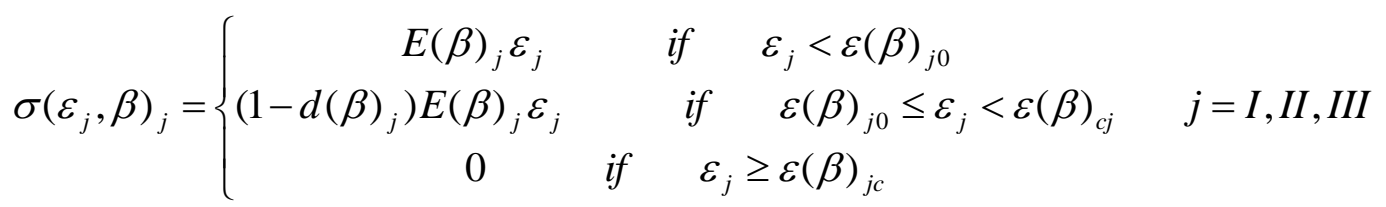


It is similar to the equ. 17 that the particle size $\beta$ cohesive was included as the length scale in the $\mathrm{CDL}$, which is part of functions for cohesive module $\mathrm{E}(\beta)_{\mathrm{j}}$, initial damage strain $\varepsilon_{\mathrm{j} 0}(\beta)$ as $\mathrm{E}(\beta)_{\mathrm{j}}=$ $\sigma_{\mathrm{jy}}(\beta) / \varepsilon_{\mathrm{j} 0}(\beta)(\mathrm{j}=\mathrm{I}, \mathrm{II}, \mathrm{III})$, critical damage strain $\varepsilon(\beta)_{\mathrm{jc}}$ and damage scale $\mathrm{d}(\beta)_{\mathrm{cj}} \cdot \sigma_{\mathrm{jy}}(\beta)$ is cohesive strength.

Obviously, equs. 17 and 18 describe two discontinue stresses fields from material inner domain to boundaries. There is no singularity problem when using these two equations to approximate the stress field in the whole domain of materials or structures even there are existing cracks. Therefore, these proposed ADL and CDL play a similar role of enrichment functions for enriching the elements containing the discontinuity of stress field. The standard Heaveside function is required to enrich the elements which are damaged, and to enrich some elements in the area with existing cracks. Damaged elements are assessed by both ADL and CDL.

In the general mixed damage mode case, two quadratic formulas given in previous work [18-19] are employed to determine the initial damage stage and final crack stage. The damage scale given in equs. 17 and 18 is proposed to be expressed by a quadratic relationship in equ. 19 .

$d=\sqrt{\left(\gamma_{I} d_{I}\right)^{2}+\left(\gamma_{I I} d_{I I}\right)^{2}+\left(\gamma_{I I I} d_{I I I}\right)^{2}}$

Each individual damage scale $d_{j}(j=I, I I$, III) in equ. 19 is used to measure the reduction of stiffness by equ. 17 and 18 in the material softening stage $\left(\varepsilon_{\mathrm{j}}>\varepsilon_{\mathrm{j} 0}\right)$. Each $\mathrm{d}_{\mathrm{j}}$ in equ. 19 can be defined by equ. 20.

$d_{j}=Q\left(1-\frac{\varepsilon_{j 0}}{\varepsilon_{j}}\right)$

Where, $\mathrm{Q}$ is a material coefficient expressed as $\varepsilon_{\mathrm{jc}} /\left(\varepsilon_{\mathrm{jc}}-\varepsilon_{\mathrm{j} 0}\right)$. Damage coupling factor $\gamma_{\mathrm{j}}$ in equ. 19 is determined as $0 \leq \gamma_{j} \leq 1.0, \quad j=I, I I, I I I$.

For the single fracture mode, for example, in opening mode, $\mathrm{j}=\mathrm{I}, \gamma_{\mathrm{I}}=1.0$ and all others are zero. Therefore, the damage scale $d=d_{I}$. For the mixed fracture mode, the value of $\gamma_{\mathrm{j}}$ is derived as follows. Firstly, assume the amount of total damage scale in mixed facture mode case equals the value of damage scale in the pure single mode I case. This can be expressed by equ. $21[18,19]$. 
$d=\sqrt{\left(\gamma_{I} d_{I}\right)^{2}+\left(\gamma_{I I} d_{I I}\right)^{2}+\left(\gamma_{I I} d_{I I I}\right)^{2}}=d_{I}$

Where, three damage coupling factors $\gamma_{\mathrm{I}}, \gamma_{\mathrm{II}}$ and $\gamma_{\mathrm{III}}$ would not be same in the general mixed damage case in reflecting different contribution from each damage mode in the total damage scale. However, $\gamma_{\mathrm{I}}, \gamma_{\mathrm{II}}$ and $\gamma_{\mathrm{III}}$ are simply treated to have a same value as $\gamma_{\mathrm{I}}=\gamma_{\mathrm{II}}=\gamma_{\mathrm{III}}=\gamma$ to simplify the problem in this investigation, and assume the mixed damage ratio is taken as $\mu=d_{I I} / d_{I}$ and $\eta=d_{I I I} / d_{I}$. Using equ. 21, $\gamma$ can be worked out by equ. 22.

$$
\gamma=\sqrt{\frac{1}{1+\mu^{2}+\eta^{2}}}
$$

The coupled effects in mixed damage mode case can be explored in a numerical iteration process. Considering a $2 \mathrm{D}$ mixed mode case $\mathrm{d}_{\mathrm{III}}=0$, taking the first order differential of equ. 21 gives the total damage rate expressed by equ. 23

$$
d^{\prime}=\frac{\gamma_{I}^{2} d_{I} A \varepsilon_{I}^{-2} \varepsilon_{I}^{\prime}(t)+\gamma_{I I}^{2} d_{I I} B \varepsilon_{I I}^{-2} \varepsilon_{I I}^{\prime}(t)}{\sqrt{\gamma_{I}^{2} d_{I}^{2}+\gamma_{I I}^{2} d_{I I}^{2}}}
$$

Where, A and B are two material constants given by equ. 24.

$$
\mathrm{A}=\frac{\varepsilon_{\mathrm{Ic}} \varepsilon_{\mathrm{I} 0}}{\varepsilon_{\mathrm{Ic}}-\varepsilon_{\mathrm{I} 0}}, \quad \mathrm{~B}=\frac{\varepsilon_{\mathrm{II}} \varepsilon_{\mathrm{II} 0}}{\varepsilon_{\mathrm{II}}-\varepsilon_{\mathrm{II} 0}}
$$

The total mixed mode damage rate in current incremental step can be expressed as

$$
d_{i+1}=\frac{\gamma_{I}^{2} d_{I i} A \varepsilon_{I i}^{-2} \varepsilon_{I(i+1)}^{\prime}(t)+\gamma_{I I}^{2} d_{I I} B \varepsilon_{I I i}^{-2} \varepsilon_{I I(i+1)}^{\prime}(t)}{\sqrt{\gamma_{I}^{2} d_{I i}^{2}+\gamma_{I I}^{2} d_{I I}^{2}}}
$$

Where, the relative displacement rate in current incremental step can be obtained by equ. 26 .

$$
\varepsilon_{j(i+1)}^{\prime}(t)=\frac{\Delta \varepsilon_{j(i+1)}}{\Delta t}, \quad j=I, I I
$$

Then, current relative displacement can be calculated by equ 27 . 


$$
\varepsilon_{j(i+1)}=\varepsilon_{j i}+\int_{\Delta t_{i}}^{\Delta t_{i+1}} \varepsilon_{j(i+1)}^{\prime} d t, \quad j=I, I I
$$

Thus equ. 20 for accounting current individual damage can be rewritten as equ. 28 .

$$
d_{j(i+1)}=Q\left(1-\frac{\varepsilon_{j 0}}{\varepsilon_{j(i+1)}}\right)
$$

Equ. 25 is a formula to account the current total damage rate in mixed mode damage case, which indicated that current damage rate of mixed damage case relates to two individual relative displacement rates in current step, two individual relative displacements and two individual damage scales in previous step. Material constants, $\varepsilon_{I 0}, \varepsilon_{I c}$ and $\gamma$ give a proportional value as a coefficient to mixed total damage rate. In single damage mode I case, $d_{I I i}=\varepsilon_{I I i}=0$, and $\gamma_{I}=1.0$, equ. 25 can be changed as

$$
d_{i+1}^{\prime}=A \frac{\varepsilon_{I(i+1)}^{\prime}(t)}{\varepsilon_{I i}^{2}}
$$

It can be seen from equ. 29 that current damage rate in single damage mode is relevant only to current relative displacement rate and previous relative displacement. It should be noticed that although $d_{I i i}=\varepsilon_{I I i}=0$ for pure mode I damage case in equ. 25 , however, the mode II related relative displacement rate $\varepsilon_{I I}^{\prime}(t)$ may not be zero. Thus, nonzero mode II related relative displacement rate would change the state of pure mode I damage to be a mixed damage case in the next step of numerical integration. Similarly, current damage rate in single damage mode II case can be given by equ. 30.

$$
d_{i+1}^{\prime}=B \frac{\varepsilon_{I I(i+1)}^{\prime}(t)}{\varepsilon_{I I i}^{2}}
$$

In numerical integration, the total damage can be accounted by equ. 31 using the damage rate.

$$
d_{j(i+1)}=d_{j i}+\int_{\Delta t_{i}}^{\Delta t_{i+1}} d_{j(i+1)}^{\prime} d t, \quad j=I, I I
$$




\section{Length scale damage analysis}

In this investigation, different micro interfacial pore sizes represented the different bonded qualities of bone-cement interface, which were determined by the physically scanned images. Figure 2 shows an image of bone-cement interface, which contains a number of pores with different sizes. Figure 2 also shows three groups of pore size at interfaces, which were catalysed as small, medium and large, and used in this investigation. These three groups of pore size were measured by XCT scanning images and their related bonding interface properties were given in Table 1. A schematic of adhesively bonded interface with particles and pores was given in Figure 3. The resin used in this investigated adhesively bonded joint is non-particles contained. The bone material usually has multiple pores. Because previous experimental work lacked the information of detailed micro construction of bone materials [3], therefore, both cement and bone were treated as non-particles contained materials in this investigation. Figure 4 shows a macroscale FEA model of bone-cement joint. The tension load was investigated in this paper. The interface between cement and bone is an adhesive layer, which was modelled by a 2D ECDM model shown in Figure 5. A micro ADL shown in Figure 6 was used in this ECDM based damage modelling. It should be noticed that three main interface properties, fracture toughness $G_{c}$, strength $\sigma$ and initial damage relative displacement $\varepsilon_{0}$, are the functions of length scale of pore size $\lambda$ to reflect the effects of pores on the interface behavior. The final damage relative displacement $\varepsilon_{\mathrm{c}}$ determined by three main interface properties in this micro ADL is also relevant to the length scale of pore size $\lambda$. Therefore, a group of interface properties with length scale of pore size played a role linking the micro ADL with the macro ECDM based FEA damage model. Interface fracture toughness $G_{c}(\lambda)$, strength $\sigma_{t}(\lambda)$ and initial damage relative displacement $\varepsilon_{0}(\lambda)$ were determined by experimental work in which three groups of pore size (small, medium and large) were investigated [3]. These varied interface properties against the length scale of pore size shown in Figure 7 were interpolated using the basic data from previous test work [3]. It should noted that the initial damage relative displacement $\varepsilon_{0}(\lambda)$ in Figure 7 was replaced by the interface initial stiffness $\mathrm{K}_{0}(\lambda)$ given by $\sigma_{t}(\lambda) / \varepsilon_{0}(\lambda)$.

It can been seen from the Figure 7 that both $\sigma_{t}(\lambda)$ and $K_{0}(\lambda)$ are decrease when length scale $\lambda$ increases from $200 \mu \mathrm{m}$ to $600 \mu \mathrm{m} . \mathrm{G}_{\mathrm{c}}(\lambda)$ increase at small lengths from $200 \mu \mathrm{m}$ and reaches the highest point at length scale $400 \mu \mathrm{m}$, then decreases at larger lengths. Comparing to $\sigma_{t}(\lambda)$ and $\mathrm{K}_{0}(\lambda)$, $\mathrm{G}_{\mathrm{c}}(\lambda)$ is less influenced by the length scale. Analytically, the functions of interface properties to length scale of pore size are given in the equ. 32 . 
$\left\{\begin{array}{l}\sigma_{t}(\lambda) \\ G_{c}(\lambda) \\ K_{0}(\lambda)\end{array}\right\}=[\mathbf{A}]\{\lambda\}=\left[\begin{array}{ccc}a_{1} & -a_{2} & -a_{3} \\ -b_{1} & b_{2} & -b_{3} \\ c_{1} & -c_{2} & c_{3}\end{array}\right]\left\{\begin{array}{c}\lambda^{2} \\ \lambda \\ 1\end{array}\right\}$

The equ. 32 established a relationship between the interface properties and length scale $[\lambda]$. The $[\mathbf{A}]$ is the material constant matrix determined by experimental data presented in Figure 7, which can be seen from equ. 33.

$[\mathbf{A}]=\left[\begin{array}{ccc}a_{1} & -a_{2} & -a_{3} \\ -b_{1} & b_{2} & -b_{3} \\ c_{1} & -c_{2} & c_{3}\end{array}\right]=\left[\begin{array}{ccc}6.125 & -10.825 & -5.34 \\ -11 & 8.4 & -0.74 \\ 9.375 & -1.525 & 12.86\end{array}\right]$

Using equ. 17, the material constitutive matrix of a $2 \mathrm{D}$ interface model given in equ. 15 can be given by equ. 34 .

$[\mathbf{D}]=\left[\begin{array}{ll}\frac{\partial \sigma_{I}}{\partial \varepsilon_{I}} & \frac{\partial \sigma_{I}}{\partial \varepsilon_{I I}} \\ \frac{\partial \sigma_{I I}}{\partial \varepsilon_{I}} & \frac{\partial \sigma_{I I}}{\partial \varepsilon_{I I}}\end{array}\right]$

In the basic bone-cement FEA model, four-noded quadrilateral plane strain elements CPE4I from ABAQUS were employed for both bone and cement, and an ECDM based user extended cohesive element in ABAQUS was used to simulate the interfacial behavior between bone and cement in this investigation. The data for geometry and materials used in this investigation were taken from the previous work [3]. A 2D mesh with dimension $10 \times 10 \mathrm{~mm}^{2}$ and unit thickness given in Figure 4 is used for both bone and cement region, which are connected by the interface. This mesh was refined with $0.2 \mathrm{~mm}$ elemental length along the interface, which was calculated according to a critical crack length $[10,18,19]$ to enable the modelling to has a convergent solution. Elastic modulus of $2 \mathrm{GPa}$ and $768 \mathrm{MPa}$ for the cement and the bone, respectively. A Poisson's ratio of 0.3 was used for both materials. It should be noticed that the behavior of adhesive layer between bone and cement was simulated by the ECDM model with zero thickness using interface fracture toughness $\mathrm{G}_{\mathrm{c}}(\lambda)$, initial stiffness $K_{0}(\lambda)$ and strength $\sigma_{c}(\lambda)$, which vary with length scale of pore size related bonding qualities at interface. Table 1 presents the properties of bone-cement interface under tension which were conducted by authors of reference 3 . 


\section{Results and comparison}

The modelling was running using ABAQUS/standard code with nonlinear displacement control. Figure 8 shows the simulated fractures of bone-cement joint under tension. The interface failure was presented by the extended cohesive user element between the bone and cement. Figure 9 shows predicted failure response together with test solution of bone-cement interface with length scale of pore size. Basically, modelling predictions had good agreement with test work in all three cases of bonding qualities. Both prediction and test show brittle failure characteristics of interface with small pore, presented by a sharp drop from the peak value in the load-separation curve. The interface with medium or large pores behaved some extent of ductility. Obviously, the interface with small pore case reached the highest value of failure load, and the large pore case had the lowest value of load capacity. The difference between predicted failure loads and tested ones in both small and medium pore cases is less than $5 \%$, but it was about $16 \%$ in large pore case due to possible errors in data collection. It should be noticed that except the difference between predicted failure loads and tested loading capacities, the predicted initial stiffness of interface under tension is lower than the measured value in all three bonding quality cases. This is possibly because the values of interface fracture energy criteria were estimated by the line fittings using the data for traction and separation which were obtained from experimental work, and the accuracy of measured interface initial damage for separation was hardly to be controlled using general mechanical measurement equipments. This error certainly affected the calculation of the interface initial stiffness used in the ECDM. This error, however, can be usually improved by standard fracture tests to obtain the better fracture energy criteria and interface strength [6]. An additional case with averaged pore was also investigated in this paper and the predicted failure response together with the test and prediction of medium pore case can be seen from Figure 10. It can be seen from Figure 10 that the prediction of averaged pore case is better than the medium pore case comparing the failure loads and initial stiffness with test solutions.

\section{Conclusions and future work}

An extended cohesive damage model (ECDM) was developed in this paper, and was used successfully in a length scale damage modelling of a bone-cement joint. In the developed ECDM, two extended damage laws were used to overcome the singularity at crack tip in stress filed. The Heaveside function was used to describe existing damages together with the standard unit partition in XFEM. The direction of damage propagation was predicted by the maximum principal stresses. The failure mechanism of bone-cement joint was investigated well comparing to experimental 
work. The effects of length scale of interfacial pores on loading capacities of bone-cement joint were successfully predicted in this investigation. The length scale of pore size at bonded interfaces played a role to link the micro damage law with macro FEA modelling. This ECDM technique supplied a potential approach to solve discontinue problems in complex materials such as adhesively bonded joints, heterogeneous adhesives and fibre composites. Future work includes length scale damage modelling of cohesive and adhesive crack propagation in ABJs under shearing and mixed mode loading, and multicrack propagation in fibre composites. During the evolution of damage propagation, the pore size would change. Future work will also consider this changing effect on the fracture behaviour of ABJs. A more general interface fracture problem with curved cracks and mixed modes to show the power of proposed ECDM will be considered in the future applications.

\section{References}

1. Xu S., Dillard, D. and Dillard J., Environmental aging effects on the durability of electrically conductive dhesive joints, Int. J. Adhes. Adhes. 2003;23:235-250.

2. Karel Matous, Mohan G. Kulkarni, Philippe H. Geubelle, Multiscale cohesive failure modeling of heterogeneous adhesives, Mechanics and Physics of Solids, 2008;56:15111533.

3. J-Y Wang, G Tozzi, J Chen, F Contal, C Lupton, J Tong, Bone-cement interfacial behaviour under mixed mode loading conditions. Mechanical Behavior of Biomedical Materials, 2010;3:392-398.

4. Clech, J.P., Keer, L.M., Lewis, J.L., A model of tension and compression cracks with cohesive zone at a bone-cement interface. J. Biomech. Eng. 1985;107:175-182.

5. Mann, K.A., Mocarski, R., Damron, L.A., Allen, M.J., Ayers, D.C., Mixedmode failure response of the cement.bone interface. J. Orthop. Res. 2001;19:1153-1161.

6. P. Moreo, M. A. Perez, J. M. Garcia-Aznar and M. Doblare, Modelling the mixed-mode failure of cement-bone interfaces, Engineering fracture mechanics, 2006;73:1379-1395.

7. J. Chen and P. J. Gregson, Application of an interface failure model to predict fatigue crack growth in an implanted metallic femoral stem, Journal of Computer Methods and Programs in Biomedicine, 2004;73(3): 249-256.

8. J. Chen, M. Crisfield, A. J. Kinloch, E. P. Busso and F. L. Matthews, Predicting progressive delamination of composite material specimens via interface elements, Mechanics of Composite Materials and Structures, 1999;6(4):301-317.

9. P. Rahulkumar, A. Jagota, S. J. Bennison and S. Saigal, Polymer interfacial fracture simulations using cohesive elements, Acta mater, 1999;47(15): 4161-4169.

10. J. Chen, Eric Ravey, Stephen Hallett and Michael Wisnom, Prediction of delamination in braided composite T-piece specimens", Composites Science and Technology, 2009;69: 2363-2367.

11. J. Chen and A. New, Application of decohesive model with mixed damage scale in fracture analysis of composite materials, Fatigue \& Fracture of Engineering Materials \& Structures, 2001;24(11):761-769.

12. Mi Y. and Crisfield M. A, Hellweg H-B and Davies G. A. O., Progressive delamination using interface elements, J. Composite Materials. 1998; 
13. Tvergaard V. and Hutchinson J. W., The influence of plasticity on mixed mode interface toughness. J. Mech. Phys. Solids, 1993;41:1119-1135.

14. Vinay K. GOYAL, Eric R. Johnson, Carlos G. Davila, Irrevesible constitutive law for modeling the delamination process using interface surface discontinuities, Composite Structures, 2004;65:289-305.

15. G. A. O. Davis, D. Hitchings, J. Ankersen, Predicting delamination and debonding in modern aerospace composite structures, Composite Science and Technology, 66, 846 -854 .

14. Robinson P, Galvanetto U, Tumino D, Bellucci G, Violeau D., 2005. Numerical simulation Of fatigue-driven delamination using interface elements. International Journal for Numerical Methods in Engineering, 2006;63:1824-1848.

15. K. L. Roe, T. Siegmund, An irreversible cohesive zone model for interface fatigue crack Growth simulation, Engineering Fracture Mechanics, 2003;70:209-232.

16. Peerlings, R. H. J., Brekelmans, W. A. M., de Borst, R., Geers, M. G. D., Graddient -enhanced damage modelling of high-cycle fatigue, Int. J. Numer. Meth. Engng. 2001;49: 1547-1569.

17. A. Turon, J. Costa, P. P. Camanho, C. G. Davila, Simulation of delamination in composites under high-cycle fatigue loading, Composites Part A, 2007;38 (11): 2270-2282.

18. J. Chen and D. Fox, Numerical Investigation Into Multi-Delamination Failure of Composite T-Piece Specimens Under Mixed Mode Loading Using A Modified Cohesive Model, Composite Structures, 2012;94:2010 - 2016.

19. J. Chen, A numerical investigation of thermal related matrix shrinkage cracking and delamination in composite T-piece specimens using a modified interface cohesive model, Thermoplastic Composite Materials, 2012;25(3): p267-282.

20. ABAQUS User Manual, Hibbitt, Karlsson \& Sorensen Inc., 1080 Main Street, RI02860 4847, USA.

21. A Ahmed and Ferdinando Auricchio, eXtended Finite Element Method(XFEM)-Modeling arbitrary discontinuities and Failure analysis, Research degree thesis, 2009, Istituto Universitario di Studi Superiori di Pavia, Universit a degli Studi di Pavia.

22. T.P. Fries, N. Moës, A. Zilian (editors), The extended finite element method, special issue, Internat. J. Numer. Methods Engrg., 2011;86:403-666.

23. $\mathrm{Xu} \mathrm{Y,} \mathrm{Yuan} \mathrm{H}$, Computational modeling of mixed-mode fatigue crack growth using extended finite element methods, Int. J. Fracture, 2009;159:151-165.

Table 1, Properties of bone-cement interface under tension

\begin{tabular}{|l|l|l|l|}
\hline $\begin{array}{l}\text { Pore } \\
\text { size }\end{array}$ & $\begin{array}{l}\text { Interface strength } \sigma_{\mathrm{t}} \\
(\mathrm{MPa})\end{array}$ & $\begin{array}{l}\text { Fracture energy } \mathrm{G}_{\mathrm{c}} \\
(\mathrm{N} / \mathrm{mm})\end{array}$ & $\begin{array}{l}\text { Initial stiffness } \mathrm{K}_{0} \\
(\mathrm{MPa} / \mathrm{mm})\end{array}$ \\
\hline small & 3.42 & 0.50 & 12.18 \\
\hline medium & 1.99 & 0.86 & 10.75 \\
\hline large & 1.05 & 0.34 & 8.57 \\
\hline
\end{tabular}




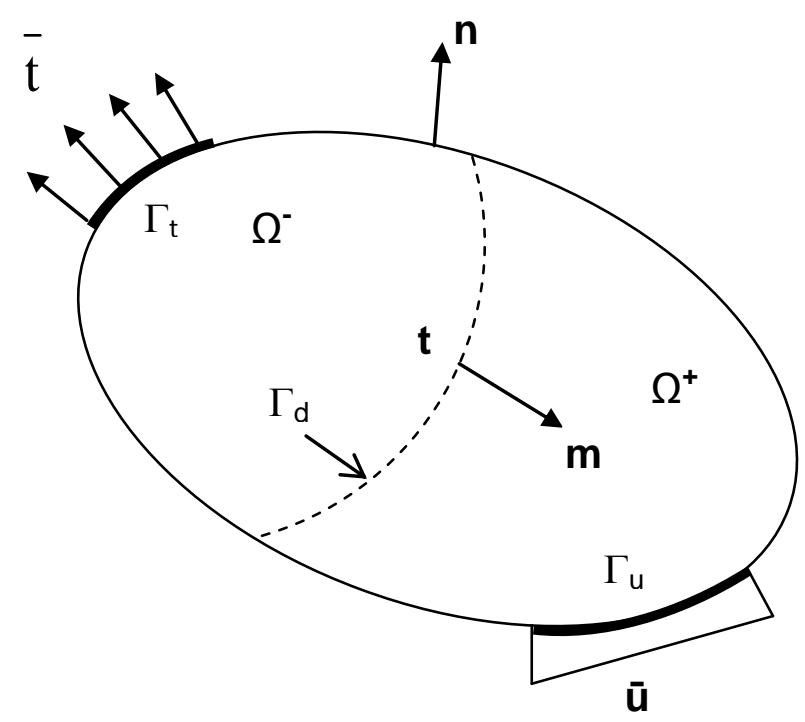

Figure 1, A body with a cohesive crack
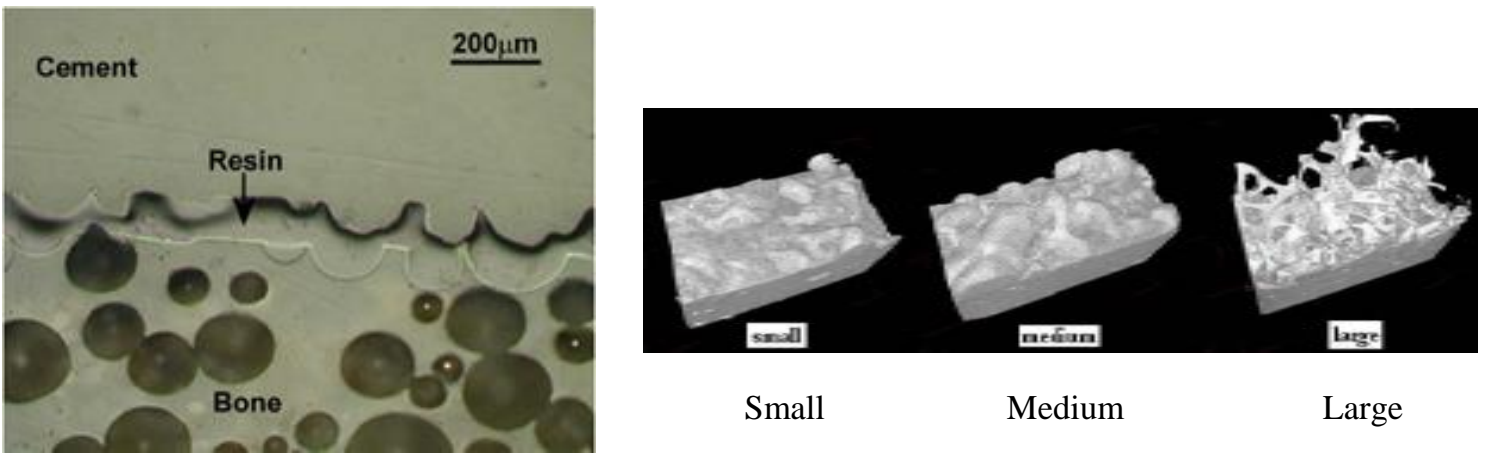

Figure 2, a. An image of bone-cement interface, b. Three groups of pore size at interface

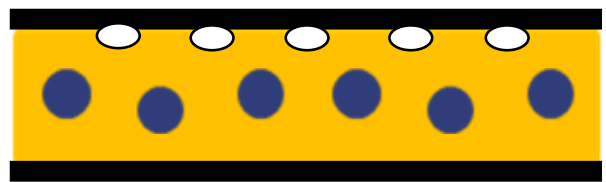

Figure 3, A schematic of adhesively bonded interface with particles (blue) and pores (white) 


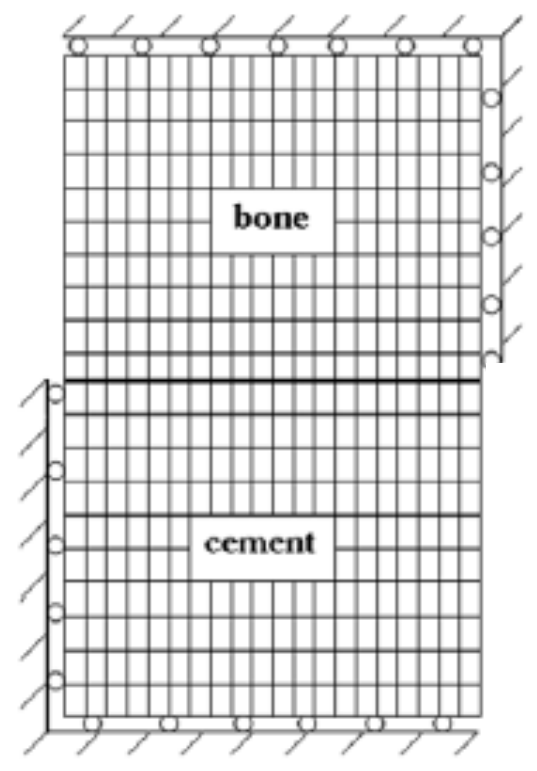

Figure 4, A macro FEA model of bone-cement joint under tension

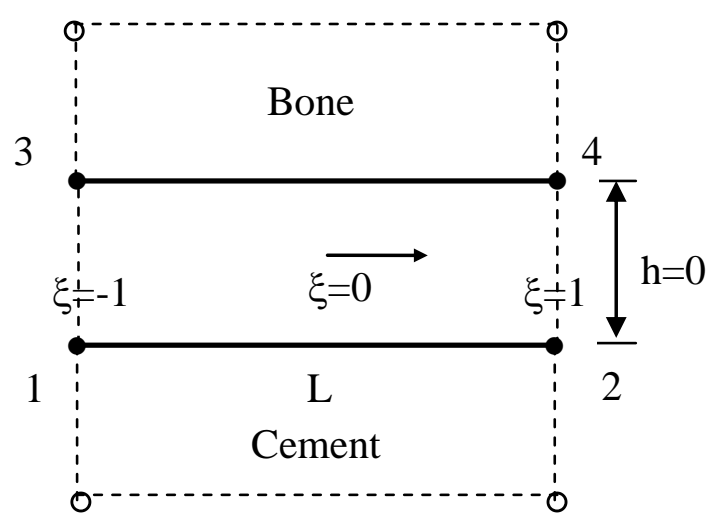

Figure 5, A linear 2D ECDM model

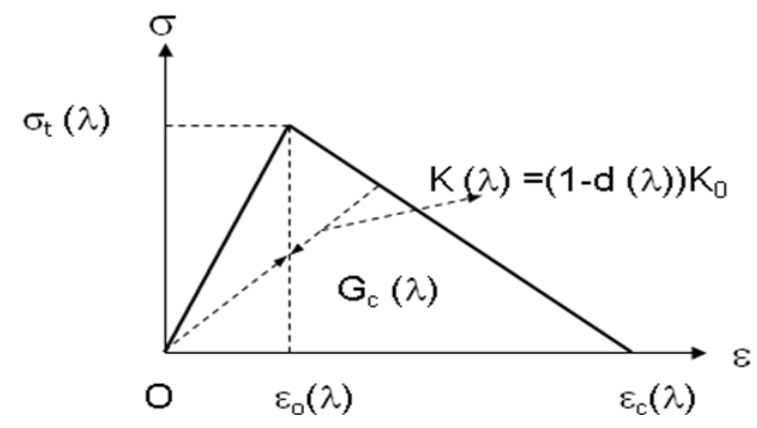

Figure 6, A micro ADL with a length scale 


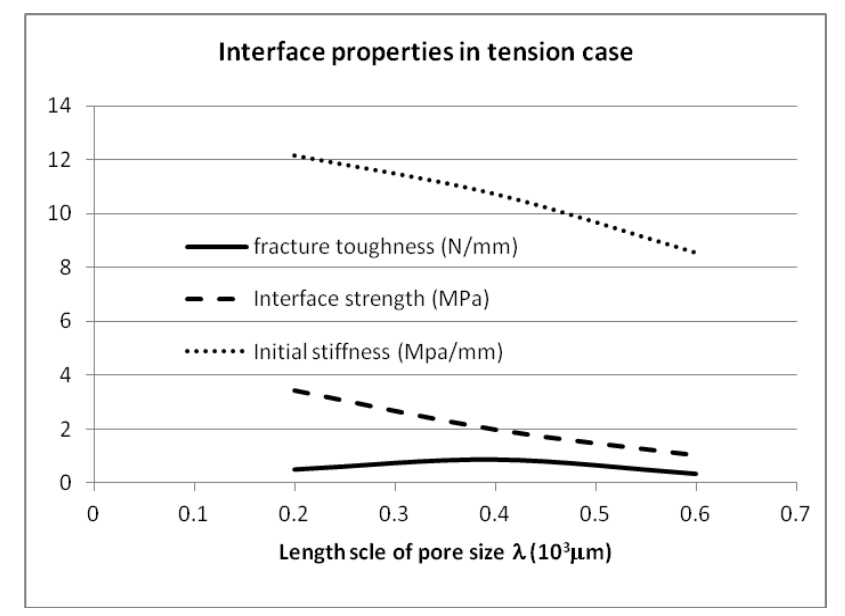

Figure 7, Interface properties against length scale of pore size



Figure 8, A bone-cement model under tension, deformed model with a. failed interface, b. removed failed interface 


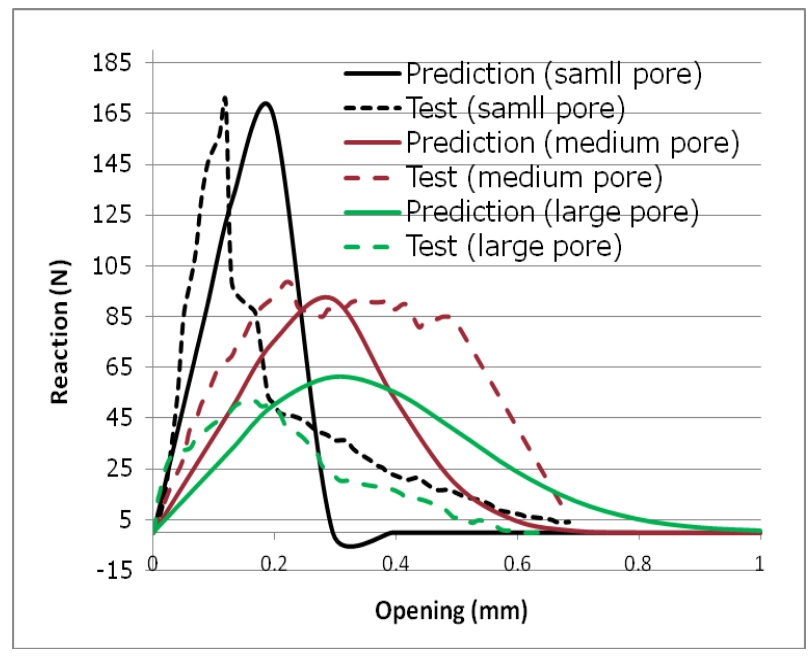

Figure 9, Failure response of interface with varied pores under tension

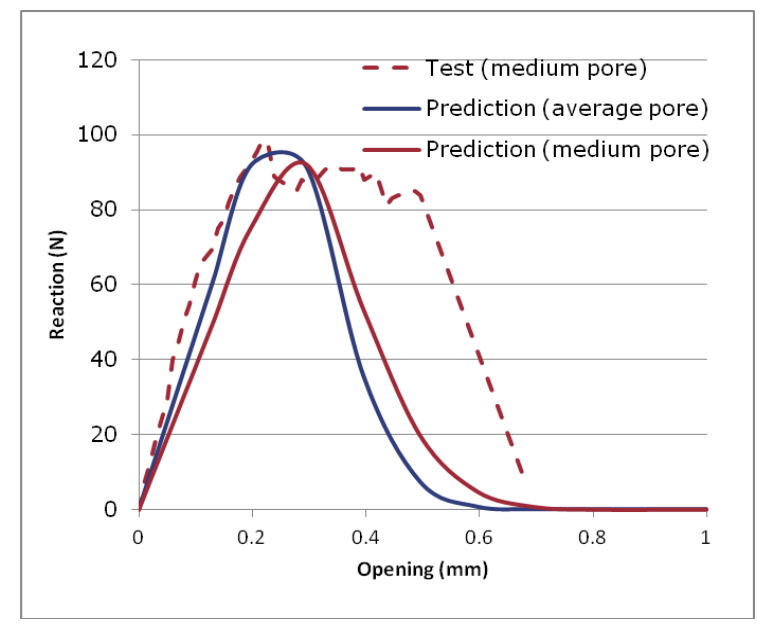

Figure 10, Failure response of interface with averaged and medium pores under tension 